\title{
On the fast coronal mass ejections in October/November 2003: ACE-SWICS results
}

\author{
Thomas H. Zurbuchen, ${ }^{1}$ G. Gloeckler, ${ }^{1,2}$ F. Ipavich, ${ }^{2}$ J. Raines, ${ }^{1}$ C. W. Smith, ${ }^{3}$ \\ and L. A. Fisk ${ }^{1}$ \\ Received 9 January 2004; revised 10 May 2004; accepted 12 May 2004; published 9 June 2004.
}

[1] Coronal Mass Ejections (CMEs) originate deep in the corona and, due to physical processes that are currently not well understood, are often accelerated to speeds well above the average solar wind speed. Solar wind compositional measurements provide important information about the source of such ejections, the heating profiles, and their expansion in the inner heliosphere. In this article we present data from the Solar Wind Ion Composition Spectrometer (SWICS), part of the Advanced Composition Explorer (ACE) spacecraft, describing the kinetic and compositional properties of the solar wind associated with CMEs that occurred in late October and early November 2003. In particular, we discuss the ionic composition of $\mathrm{C}, \mathrm{O}$, and $\mathrm{Fe}$, as well as the relative elemental composition of $\mathrm{C}, \mathrm{O}$, and $\mathrm{Fe}$, present in those CMEs. INDEX TERMS: 2164 Interplanetary Physics: Solar wind plasma; 7513 Solar Physics, Astrophysics, and Astronomy: Coronal mass ejections; 7815 Space Plasma Physics: Electrostatic structures; 7899 Space Plasma Physics: General or miscellaneous. Citation: Zurbuchen, T. H., G. Gloeckler, F. Ipavich, J. Raines, C. W. Smith, and L. A. Fisk (2004), On the fast coronal mass ejections in October/November 2003: ACE-SWICS results, Geophys. Res. Lett., 31, L11805, doi:10.1029/2004GL019461.

\section{Introduction}

[2] Coronal Mass Ejections (CMEs) are transient events in which large quantities of plasma are ejected from the corona into the heliosphere [Gosling et al., 1974; Hundhausen, 1993]. These ejections typically originate in closed-field regions in the corona, are often associated with solar-active regions, and have a magnetic field topology that is highly complex. The ejections of mass from the Sun are also often associated with solar flares [Cane, 1997, and references therein]. Identification of ICMEs (Interplanetary CMEs) is based on several characteristics, including a strong magnetic field, rotating magnetic field ("fluxrope") signatures, unusually low proton and electron temperatures, bidirectional electron signatures, and compositional signatures such as unusually high $\mathrm{He} / \mathrm{H}$, elevated Fe charge states, and unusually high charge states of C and O [Burlaga et al., 1981; Gosling, 1990, 1997; Zurbuchen et al., 2003; Henke et al.,

\footnotetext{
${ }^{1}$ Department of Atmospheric, Oceanic, and Space Sciences, University of Michigan, Ann Arbor, Michigan, USA.

${ }^{2}$ Department of Physics, University of Maryland, College Park, Maryland, USA.

${ }^{3}$ Department of Physics and Institute for the Study of Earth, Oceans and Space, University of New Hampshire, Durham, New Hampshire, USA.

Copyright 2004 by the American Geophysical Union. 0094-8276/04/2004GL019461
}

1998; Lepri et al., 2001]. Any given ICME may possess some or all of these signatures, but no one signature is present in all ICMEs. Moreover, ICMEs may display different timings for these signatures so that exact begin and end times for the ejecta can be ambiguous.

[3] The elemental composition of the solar wind is generally ordered according to the first ionization potential (FIP). Elements with FIP $<10 \mathrm{eV}$ are generally enhanced over the other elements [von Steiger et al., 2000]. The level of enhancement of low-FIP elements strongly depends on the solar wind source region. Fast coronal-hole-associated solar wind is less fractionated than slow solar wind associated with streamers or loops in the corona. In general, ICMEs have elemental composition signatures similar to slow solar wind [Zurbuchen et al., 2003]. One major exception to this rule is the He abundance. There are well-known enhancements of $\mathrm{He} / \mathrm{H}$ and $\mathrm{He} / \mathrm{O}$ associated with many ICMEs. Generally, the enhancements only cover a small fraction of the total volume of the ICMEs [Borrini et al., 1982]. There are also a few reports of unusual, strongly mass-dependent elemental enhancements in some unique ICMEs [Gloeckler et al., 1999; Wurz et al., 1998].

[4] Even though there are similarities between the elemental composition of ICMEs and slow solar wind, there are remarkable differences in the ionization states of ICMEs from the average ionic composition of fast and slow wind. In a few events, unusually low ionization states are found [e.g., Skoug et al., 1999, and references therein]. Such "cold charge states" are relatively rare. Most other ICMEs have charge states that indicate source temperatures greater than or equal to that of the average solar wind. Approximately half of all ICMEs have elevated charge states of all observed heavy elements, most notably $\mathrm{C}, \mathrm{O}$, and Fe. Indeed, $\mathrm{Fe}$ charge state enhancements have proven to be one of the most reliable ICME identifiers [Lepri et al., 2001]. The observed elevated charge states indicate heating of the ICME plasma to temperatures $>4 \mathrm{MK}$, far above average solar wind freeze-in temperatures, which are around $1.5 \mathrm{MK}$.

\section{The SWICS Instrument}

[5] Observations reported here, as those in many extreme ICMEs, are at the limit of what is observable by modern solar wind plasma instruments. The unique configuration of SWICS [for details, refer to Gloeckler et al., 1998] makes it possible to overcome all measurement challenges associated with these very fast ICMEs.

[6] SWICS measures solar wind ions from 0.2 to $86 \mathrm{keV} / \mathrm{q}$. The upper energy limit corresponds to a speed of $4062 \mathrm{~km} / \mathrm{s}$ for protons and $2872 \mathrm{~km} / \mathrm{s}$ for $\mathrm{He}^{2+}$. SWICS allows for an 

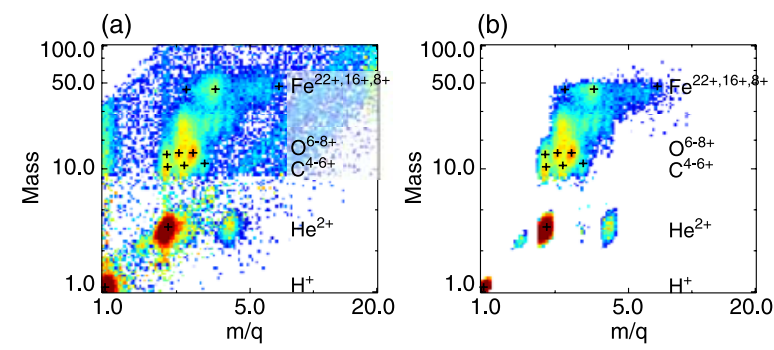

Figure 1. Solar wind mass versus mass/charge scatter plot during highly perturbed solar wind conditions on 29 October 2003. Panel (a) shows raw data during highly elevated background conditions, high solar wind fluxes, and large background energetic particle fluxes, causing both random coincidences and false times-of-flight. Panel (b) shows the same data with background suppression and overlap removal through use of the triple coincidences (provided by SWICS) and off-line processing. The locations of some solar wind ions are indicated with black crosses.

unambiguous measurement of particle speed, $V$, mass, $M$, and charge, $Q$, in triple coincidence. Figure 1a shows raw data from 29 October 2003 to demonstrate the background effect from these fast ICMEs. Nearly all background is eliminated when adding information on the energy/charge distributions of these ions and performing probabilistic assignment for peak overlap removal [von Steiger et al., 2000], as shown in Figure 1b. In summary, SWICS is ideally suited to analyze the composition of high-speed solar wind, even with large background conditions.

\section{Observations}

[7] We focus here on solar wind observations from October 28 (day-of-year 301) to November 6 (day-of-year 310), 2003. This time period includes two of the fastest ICMEs ever measured in the solar wind. The plasma and field properties of these ICMEs are discussed in detail by Skoug et al. (R. M. Skoug et al., Extremely high-speed solar wind: October 29-30, 2003, submitted to the Journal of Geophysical Research, 2004, hereinafter referred to as Skoug et al., submitted manuscript, 2004). Figure 2 shows the speed, temperature, and density of solar wind alpha particles measured during this interval with a 12-minute time resolution. The speed of solar wind alpha particles generally corresponds to the speed of solar wind protons to within $<10 \%$ [von Steiger et al., 2000]. The kinetic properties of the alpha particles were measured by SWICS without the need for extrapolation [Skoug et al., submitted manuscript, 2004]. We have also plotted 1-minute averages of the ACE magnetic field data. The 8-day time period contained at least three disturbances driven by fast ICMEs. ACE encountered two of these ICMEs but missed the third one, which passed to the west of ACE (J. T. Gosling, private communication, 2004). These disturbances produced the fastest solar wind speeds yet measured directly and drove shocks identified in the ACE magnetic field data [Cliver et al., 1990; Skoug et al., submitted manuscript, 2004]. The highest speeds occurred in the sheath regions immediately downstream from those shocks and not within the ICMEs. The maximum speed on October 29th was $1900 \mathrm{~km} / \mathrm{s}$; the largest speed associated with the second ejection, late October 30th, was $1940 \mathrm{~km} / \mathrm{s}$. The third ICME had an associated maximum speed of $772 \mathrm{~km} / \mathrm{s}$, again measured in the sheath of an ICME which ACE never actually encountered. The first two ICMEs were encountered by ACE and were apparently the fastest ICMEs observed in situ during this solar cycle [Cane and Richardson, 2003]. Assuming a constant $\mathrm{He} / \mathrm{H}$ ratio, we can calculate the dynamic pressure increase for the two fastest ICMEs based on the alpha particle measurements. Under this assumption, the dynamic pressure increase at the ICME-driven shocks was approximately a factor of 18 for the first ICME, and a factor of 5 for the second ICME.

[8] The second impressive feature of these shocks was the heating of $\mathrm{He}$, at their maximum exceeding commonly observed values by over one order of magnitude. At the first shock, the He temperature increased by over a factor of 10 , and at the second shock by over a factor of 25. Also, it is evident from Figure 2 that the magnetic field strength increased by approximately a factor of 3 and a factor of 7 at these two shocks. Meanwhile, the solar wind alpha particle density only increased by a factor of $<2$ for the first shock, and had little or no increase at the second shock. This possibly indicates a highly structured shock geometry and/or magnetic field draping about the ejecta, and will be the subject of a future investigation.

[9] The ionic charge states of $\mathrm{C}, \mathrm{O}$, and $\mathrm{Fe}$ as a function of time are shown in Figure 3. Each data point is a two-hour average of the average charge states. This ensures $<10 \%$ statistical error throughout the entire time interval. Figure 4 shows detailed charge spectra for representative two-hour intervals during this time period. On October 28, before the arrival of the first fast disturbance, the $\mathrm{C}, \mathrm{O}$, and $\mathrm{Fe}$ ionization states were consistent with a source that was characteristic of slow solar wind, $\sim 1.5 \mathrm{MK}$. The dominant charge states were $\mathrm{C}^{5+}, \mathrm{O}^{6+}$, and $\mathrm{Fe}^{9+}$, respectively. This is consistent with long-term averages of slow solar wind

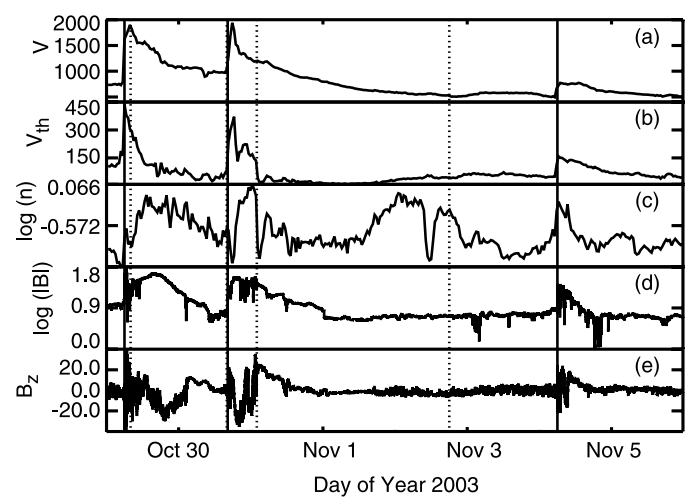

Figure 2. Solar wind plasma and field properties. From top to bottom, panels show the (a) alpha speed in $\mathrm{km} / \mathrm{s}$, (b) thermal speed in $\mathrm{km} / \mathrm{s}$ and (c) density in $\mathrm{cm}^{-3}$, (d) magnetic field magnitude in $\mathrm{nT}$, and (e) the z-component of the magnetic field in GSE coordinates, again in nT. Solid vertical lines indicate shocks identified by the magnetic field data, dashed lines indicate ICME start and end times based on plasma data [Skoug et al., submitted manuscript, 2004]. ACE did not encounter the ICME driving the disturbance and shock around November 4th. 


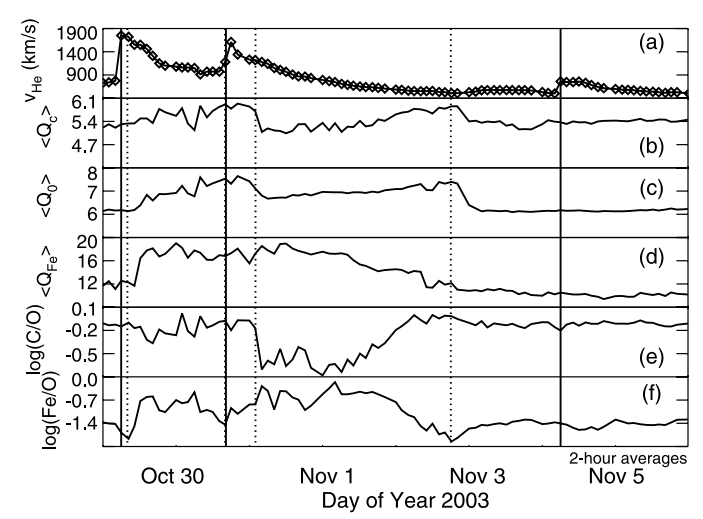

Figure 3. Composition of $\mathrm{C}, \mathrm{O}$, and Fe. From top to bottom we show two-hour averages of helium speed, and the average charge state of $\mathrm{C}, \mathrm{O}$, and $\mathrm{Fe}, \mathrm{C} / \mathrm{O}$, and $\mathrm{Fe} / \mathrm{O}$ as a function of time. The second, fast ICME shows a clearly anomalous elemental composition. Solid vertical lines indicate shocks identified by the magnetic field data, dashed lines indicate ICME start and end times based on plasma data [Skoug et al., submitted manuscript, 2004]. For more discussion, refer to text.

associated with streamers [von Steiger et al., 2000]. The first fast ICME was marked by an abrupt transition to higher charge states (higher freezing-in temperature) clearly seen in $\mathrm{O}$ and $\mathrm{Fe}$, but not so well in $\mathrm{C}$. These unusually high charge states persisted for more than one day. During one half-day, the dominant charge states were fully ionized $\mathrm{C}, \mathrm{O}$, and $\mathrm{Ne}$ like $\mathrm{Fe}^{16+}$ (as shown during instances 4 and 5 in Figure 4); the Fe charge distribution still had significant contributions above charge state 20. On October 30, the second shock arrived, propagating into the first, unusually hot ICME. It was driven by an ejecta that had lower ionization states than the first ICME, but still much higher than that of the average solar wind. The transition into this second ejecta was much more abrupt in $\mathrm{C}$ and $\mathrm{O}$ than in $\mathrm{Fe}$. The $\mathrm{Fe}$ ionization state gradually relaxed to a cooler (lower charge state), more average solar wind-like composition, until the third disturbance arrived. The $\mathrm{C}$ and $\mathrm{O}$ ionization states first increased, as observed in the previous ICME, and then dropped to low, solar wind-type composition. There were obvious differences between the ionic composition of the two ICMEs, which might be expected if the ICMEs had distinctly different thermal histories. $\mathrm{C}$ and $\mathrm{O}$ charge states freeze-in at high densities and thus measure the freeze-in temperature in the lower corona; $\mathrm{Fe}$ freezes in at low densities at 3-5 solar radii [Bürgi and Geiss, 1986]. Differences between these charge states can therefore result from temporal dependences of the ICME heating rates, or from differences in the expansion profiles of solar wind and CMEs in the low corona.

[10] Figures $3 \mathrm{e}$ and $3 \mathrm{f}$ show the elemental composition during these events. We show $\mathrm{C} / \mathrm{O}$ and $\mathrm{Fe} / \mathrm{O}$. Most ICME start and stop times, indicated by dashed lines, also have abrupt transitions of the ionization states, identifying distinct plasmas. The sharp transitions in the charge states of the ions were sometimes accompanied by distinct transitions in the elemental composition. This was most evident for the second fast ejection, where $\mathrm{C} / \mathrm{O}$ decreased by more than a factor of 2, while $\mathrm{Fe} / \mathrm{O}$ increased by more than a factor of 3 relative to their respective pre-ICME values. Meanwhile, the first and third fast ICMEs under consideration showed little change relative to average solar wind composition.

\section{Discussion}

[11] The observations during this nine-day interval include two ICMEs associated with the fastest ICMErelated disturbances ever reliably observed in situ. Both ICMEs had disturbances with plasma reaching speeds in excess of $1900 \mathrm{~km} / \mathrm{s}$. Due to the superb background suppression properties of the SWICS sensor, the solar wind compositional changes can be inferred from these data throughout the entire time interval. The shocks associated with these events are interesting because, at least in the first shock, the increase in magnetic field strength appears to have been larger than would be expected from the increase in alpha particle density. It may be that the large speed of the first fast ICME produced a significant draping of the magnetic field ahead of it, causing a larger than expected increase in magnetic field strength. This will be explored in detail in an upcoming study.

[12] All ICMEs in this time period had unusually high charge state compositions with an average Fe charge state in excess of 18 for the first and second fast ICMEs. Under the assumption of collisional equilibrium, these charge distributions can be achieved only at electron temperatures in excess of $8 \mathrm{MK}$. The high charge states imply high electron temperatures in the corona, and thus relatively high electron pressure. It is tempting to suggest that the high pressure was the cause of the unusually large speeds, although detailed model simulations would be required for a conclusive argument. It is also possible that the extreme coronal electron temperatures were simply an indication of the strong release of magnetic energy, or that in these fast events the magnetic field configuration was such that there
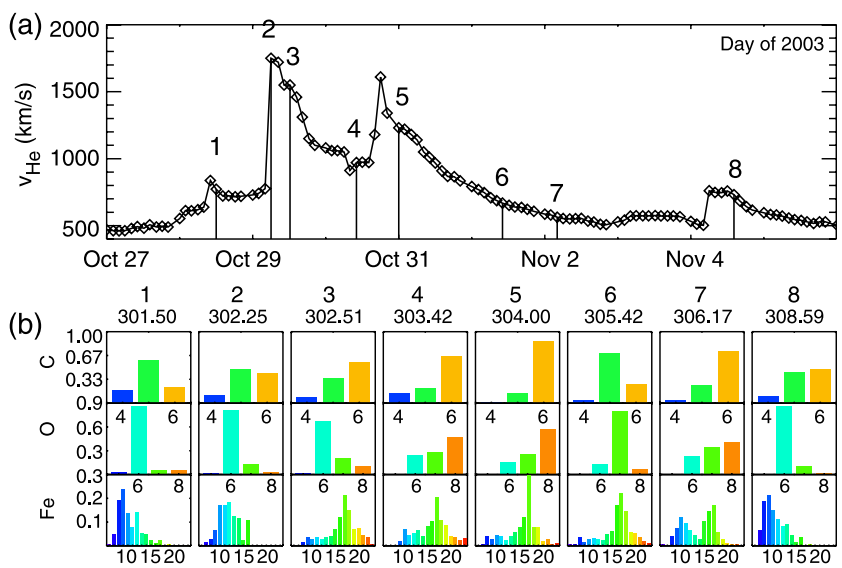

Figure 4. Charge distributions of $\mathrm{C}, \mathrm{O}$, and $\mathrm{Fe}$ during representative two-hour periods. The top panel (a) shows the speed of the alpha particles and marks representative times for which $\mathrm{C}, \mathrm{O}$, and $\mathrm{Fe}$ charge distributions are given (b). The distributions show large variations of freeze-in conditions between $\sim 1 \mathrm{MK}$ and $>5 \mathrm{MK}$. (The colors simply distinguish among contributions by individual charge states.) 
was a direct connection of this CME material to the flares that were observed, e.g., from RHESSI (R. B. Lin, private communication, 2004).

[13] The transition from the first to second fast ICME is particularly interesting. The charge compositions of elements that freeze in at high density $(\mathrm{C}, \mathrm{O})$ changed abruptly there. However, the Fe charge composition, which freezes-in in the outer corona, hardly changed there. This is unusual high $\mathrm{C}, \mathrm{O}$ freeze-in temperatures usually accompany high $\mathrm{Fe}$ freeze-in temperatures. We suggest that our observations provide evidence for plasma interactions between the two fastest ICMEs, perhaps through reconnection. This process thermally linked two individual CMEs in the outer corona.

[14] The second fast ICME had a highly unusual elemental composition, with a large decrease of $\mathrm{C} / \mathrm{O}$, and a sharp but small increase in $\mathrm{Fe} / \mathrm{O}$. Qualitatively similar signatures have been reported by Wurz et al. [1998] for another ICME. It should be possible to identify the source of these $\mathrm{C} / \mathrm{O}$ decreases, by studying the EUV, X-ray, and gamma-ray signatures of these flares, and relating the observed in situ plasma to its source. The reasons for such changes in elemental composition are not known, but the compositional signatures should yield important clues to the physics of the solar corona and its coronal mass ejections.

[15] Acknowledgments. This work was supported, in part, by NASA contract NAG 5-6921. We thank the entire SWICS team [see Gloeckler et al., 1998] for their efforts in developing this instrument. We acknowledge helpful comments from J. T. Gosling and an anonymous referee.

\section{References}

Borrini, G., J. T. Gosling, S. J. Bame, and W. C. Feldman (1982), Helium abundance enhancements in the solar wind, J. Geophys. Res., 87, 7370.

Bürgi, A., and J. Geiss (1986), Helium and minor ions in the corona and solar wind - dynamics and charge states, Solar Phys., 103, 347.

Burlaga, L., E. Sittler, F. Mariani, and R. Schwenn (1981), Magnetic loop behind an interplanetary shock: Voyager, Helios, and IMP 8 observations, J. Geophys. Res., 86, 6673.

Cane, H. V. (1997), The current status in our understanding of energetic particles, coronal mass ejections, and flares, in Coronal Mass Ejections, Geophys. Monogr. Ser., vol. 99, edited by N. Crooker, J.-A. Joselyn, and J. Feynman, p. 205, AGU, Washington, D.C.
Cane, H. V., and I. G. Richardson (2003), Interplanetary coronal mass ejections in the near-Earth solar wind during 1996-2002, J. Geophys. Res., 108, 1156, doi:10.1029/2002JA009817.

Cliver, E. W., J. Feynman, and H. B. Garrett (1990), An estimate of the maximum speed of the solar wind, 1938-1989, J. Geophys. Res., 96, 17,103 .

Gloeckler, G., et al. (1998), Investigation of the composition of solar and interstellar matter using solar wind and pickup ion measurements with SWICS and SWIMS on the ACE spacecraft, Space Sci. Rev., 86, 495537.

Gloeckler, G., et al. (1999), Unusual composition of the solar wind in the 2-3 May 1998 CME observed with SWICS on ACE, Geophys. Res. Lett., 26, 157.

Gosling, J. T. (1990), Coronal mass ejections and magnetic flux ropes in interplanetary space, in Physics of Magnetic Flux Ropes, edited by C. T. Russell, E. R. Priest, and L. C. Lee, p. 343, AGU, Washington, D.C.

Gosling, J. T. (1997), Coronal mass ejections: An overview, in Coronal Mass Ejections, Geophys. Monogr. Ser., vol. 99, edited by N. Crooker, J.-A. Joselyn, and J. Feynman, p. 9, AGU, Washington, D.C.

Gosling, J. T., et al. (1974, Mass ejections from the Sun: A view from Skylab, J. Geophys. Res., 79, 4581.

Henke, T., et al. (1998), Differences in the $\mathrm{O}^{7+} / \mathrm{O}^{6+}$ ratio of magnetic cloud and non-cloud coronal mass ejections, Geophys. Res. Lett., 25, 3465.

Hundhausen, A. J. (1993), Sizes and locations of CMEs: SMM observations from 1980-1989, J. Geophys. Res., 98, 13,177.

Lepri, S. T., T. H. Zurbuchen, L. A. Fisk, I. G. Richardson, H. V. Cane, and G. Gloeckler (2001), Iron charge distribution as an identifier of interplanetary coronal mass ejections, J. Geophys. Res., 106, 29,231.

Skoug, R. M., et al. (1999), A prolonged $\mathrm{He}^{+}$enhancement within a coronal mass ejection in the solar wind, Geophys. Res. Lett., 26, 161.

von Steiger, R., et al. (2000), Composition of quasi-stationary solar wind flows from Ulysses/Solar Wind Ion Composition Spectrometer, J. Geophys. Res., 105, 27,217.

Wurz, P., et al. (1998), Elemental composition of the January 6, 1997 CME, Geophys. Res. Lett., 25, 2557.

Zurbuchen, T. H., L. A. Fisk, S. T. Lepri, and R. von Steiger (2003), The composition of interplanetary mass ejections, in Solar Wind 10, AIP Proc., vol. 679, edited by M. Velli, R. Bruno, and F. Malara, p. 604, Melville, New York.

L. A. Fisk, G. Gloeckler, J. Raines, and T. H. Zurbuchen, Department of Atmospheric, Oceanic, and Space Sciences, University of Michigan, Ann Arbor, MI, USA. (thomasz@umich.edu)

F. Ipavich, Department of Physics, University of Maryland, College Park, MA, USA.

C. W. Smith, Department of Physics and Institute for the Study of Earth, Oceans and Space, University of New Hampshire, Durham, NH, USA. 